\title{
ZUGANG ZU REGISTER- UND INDIVIDUALDATEN FÜR DIE WISSENSCHAFTLICHE FORSCHUNG IN ÖSTERREICH
}

GERHARD SCHWARZ, THOMAS KÖNIG, HARALD OBERHOFER UND MICHAEL STRASSNIG

DOI: $10.22163 /$ fteval.2020.464

\section{EINLEITUNG}

$\mathrm{E}$ ine zentrale Aufgabe öffentlicher Verwaltung besteht darin, Datenbestände anzulegen - zur Erfüllung ihrer ureigensten Aufgaben, aber auch zur Nutzung durch andere. Diese Datenbestände dienen als Entscheidungsgrundlage, zur Planung, Dokumentation, Kontrolle und Evaluierung. So entstehen Register: von der Todesursachenstatistik über die Sozialversicherungsdaten und die Transparenzdatenbank bis zur Statistik Austria, dem größten österreichischen "Datenbunker".

Der Zugang zu vielen hochwertigen Datensätzen ist in der Regel stark eingeschränkt, oder gar nicht möglich. ' Dabei geht es um die Wahrung des Datenschutzes bzw. der Geheimhaltungsbedürfnisse der in den Registern erfassten natürlichen und juristischen Personen. Es liegt aber auch nicht unbedingt im Eigeninteresse der Bürokratie, Daten nach außen zu geben, die gegebenenfalls politisch sensibel sind. Ein besonders anschauliches Beispiel publizierte die New York Times kürzlich in Bezug auf ihre Recherchen zu den EU-Agrarsubventionen:

The European Union, we learned, maintains a database with all this information. But when we requested it, officials responded both that the data did not exist and that it did exist but could not easily be extracted. When we pointed out that the European Union had previously managed to extract the information for a World Bank study, they said the data was private. We appealed. We were denied again. Downloading the information from the government's own computers was so complicated, officials said, that they could not be forced to do it. (Apuzo 2019)

Jedoch: Hat die Öffentlichkeit nicht das Recht, über die Verwendung von staatlichen Mitteln Bescheid zu wissen? Und ist es nicht die Pflicht einer öffentlichen Verwaltung in einer demokratischen Gesellschaft, ihre Datensätze zur Analyse auf Basis von wissenschaftlichen, nachvollziehbaren Kriterien bereitzustellen, um evidenzbasierte Aussagen über staatliches bzw. hoheitliches Handeln zu erlauben und damit zu verbessern? Dies wäre zumindest im Rahmen einer politischen Diskussion über Nutzen und Risiken der wissenschaftlichen Verwendung von solchen Daten zu berücksichtigen - eine Diskussion, die aber bisher noch nicht geführt wurde. In einigen europäischen Ländern ist dies inzwischen auch passiert, und wenn auch die Grenze von Land zu Land verschieden gezogen wurde, so gilt für diese Fälle, dass sie dazu eine gültige Rechtslage hergestellt haben. Auch in Österreich hat sich dazu in den letzten Jahren etwas getan. Der vorliegende Artikel liefert dazu einen konzisen Überblick.

\section{DATENZUGANG - STAND DER DINGE IN ÖSTERREICH}

Zunächst: Die Hürden, mit denen die Journalisten der New York Times in Brüssel zu kämpfen hatten, sind den Wissenschafterinnen und Wissenschafter in Österreich nur zu vertraut. Ein Beispiel betrifft die derzeit in Diskussion befindliche Forschungsfinanzierungsdatenbank (FFDB). Hier gibt es Vorbehalte, der Wissenschaft Zugang zu pseudonymisierten Daten der staatlichen Forschungsförderung auf Unternehmensebene zu gewähren.

Wie politisch heikel der Zugang der Wissenschaft zu öffentlichen Datenbeständen gesehen wird, zeigt auch das Forschungsorganisationsgesetz (FOG). Mit dem „Datenschutz-Anpassungsgesetz 2018 - Wissenschaft und Forschung "2 ${ }^{2}$ wurde im FOG ein Rechtsanspruch wissenschaftlicher Einrichtungen auf Bereitstellung von Daten aus bundesgesetzlich vorgesehen Registern geschaffen ${ }^{3}$. Dies war ein großer Erfolg des Bundesministeriums für Bildung, Wissenschaft und Forschung (BMBWF),

Eine Ausnahme bilden Informationen des öffentlichen Sektors (PSI), die bereits über Open Data Österreich (https://www.data.gv.at/) frei zugänglich sind. Oft handelt es sich dabei um Datensätze, die eine bestimmte Anwendungsrelevanz haben, aber für eine wissenschaftliche Nutzung nur von beschränktem Wert sind.

2 BGBI. I Nr. $31 / 2018$

3 Einen Überblick zu den Vorzügen und Herausforderungen der Nutzung administrativer Daten in den Sozialwissenschaften bieten Connelly-Dibbend-GaylecPlayford. (2016). 
das sich vehement für die Bereitstellung von Registerdaten für die Wissenschaft eingesetzt hat. Wie umstritten dieses Gesetz war, zeigen die knapp 80 Stellungnahmen, die für die parlamentarische Begutachtung von vielen Stakeholdern eingegangen sind. Neben Kritik am Gesetzesentwurf wurde insbesondere von der Wissenschaftsseite auf die Wichtigkeit für den Forschungsstandort hingewiesen. ${ }^{4}$

Leider kommt dieser wichtige Erfolg für die Wissenschaft mit zwei Wermutstropfen. Erstens haben spezifischere Regelungen zum Datenschutz und zur wissenschaftlichen Datenverarbeitung in Materiengesetzen Vorrang vor den Bestimmungen des FOG. Praktisch führt dies zu komplexen rechtsdogmatischen Diskussionen über die anzuwendenden Regelungen. So deutet die derzeitige Rechtsprechung der Datenschutzbehörde (DSB) darauf hin, dass sie - anders als die Erläuterungen zur Regierungsvorlage zu den einschlägigen Bestimmungen des FOG - keinen Vorrang der Bestimmungen des FOG gegenüber jenen des Datenschutzgesetzes (DSG) sieht. Endgültig entscheiden werden dies erst die Höchstgerichte.

Zweitens müssen Register, wenn die Bestimmungen des FOG anwendbar sind, erst einzeln mittels einer gemeinsamen Verordnung der/ des zuständigen Ressortministers/in und des/der Bundesministers/in für Bildung, Wissenschaft und Forschung bereitgestellt werden. Damit bleibt es weiterhin den Ressorts vorbehalten, zu entscheiden, ob und welche bundesgesetzlich vorgesehenen Register der Wissenschaft zur Verfügung stehen. Auch angesichts der Vielzahl von für die Forschung relevanten Registern wird dies ein langwieriger Prozess sein.

\section{VERHEDDERT IM REGELUNGSDSCHUNGEL}

So wichtig die Implementierung der Registerforschung im FOG für die Wissenschaften in Österreich und ihren Beitrag zur "good governance" auch ist, so ist es damit leider nicht gelungen, eine einheitliche Regelung des Zugangs der Wissenschaft zu den Mikrodatenbeständen der öffentlichen Hand zu schaffen. Register, die unmittelbar auf EU-Verordnungen oder Landesgesetzen beruhen, sind nicht vom FOG erfasst, das sich schon aus Kompetenzgründen auf bundesgesetzlich vorgesehene Register beschränkt. Ob und wann auch die Bundesländer ihre Register für die Wissenschaft öffnen werden, steht in den Sternen.

Parallel zur Regelung der Registerforschung im FOG bestehen bzW. entstehen weitere Regelungskorpusse für große und forschungsrelevante österreichisches Datenbestände. So wurde im Transparenzdatenbankgesetz (TDBG) die Möglichkeit geschaffen ${ }^{5}$, Daten aus der Transparenzdatenbank, die die Einkommen und erhaltenen öffentlichen Leistungen aller natürlichen und juristischen Personen in Österreich enthält, „in anonymisierter Form an fachlich geeignete Personen oder wissenschaft- liche Einrichtungen für wissenschaftliche Zwecke nach Vereinbarung der konkreten Anwendungsbereiche zu übermitteln" (§ 34 Abs. 2 TDBG). Bemerkenswert daran ist einerseits, dass der Bundesminister für Finanzen nach dem Wortlaut der Bestimmung zwar verpflichtet ist ${ }^{6}$, die Daten für wissenschaftliche Verwendung zu übermitteln, allerdings nur zur Erfüllung der in § 2 TDBG genannten Zwecke ${ }^{7}$. Ob ein solcher Anspruch in der Praxis tatsächlich rechtlich durchsetzbar wäre, bleibt fraglich.

Andererseits ist an der Regelung der Datenübermittlung für wissenschaftliche Zwecke im TDBG bemerkenswert, dass sich diese nur auf „anonymisierte“ Daten bezieht. Somit ist der Zugriff der Wissenschaft auf grob auflösende Daten beschränkt, da ansonsten die Identifikation einzelner Merkmalsträger anhand bestimmter Ausprägungen von Merkmalen in den Daten nicht ausgeschlossen werden kann ${ }^{8}$. Es bedeutet jedoch auch, dass die Daten keine Schlüsselvariablen enthalten dürfen, die eine Verknüpfung mit anderen Datenbeständen ermöglichen würde ${ }^{9}$, da solche Daten nur "pseudonymisiert", aber nicht "anonymisiert" sind. Die Verknüpfung von Daten aus der Transparenzdatenbank mit anderen Datenbeständen zu Zwecken der Auswertung bleibt ausschließlich der "Statistik Austria“ vorbehalten (§ 18 Abs. 3 TDBG). Grob auflösende Daten und die Nichtverknüpfbarkeit sind aber ein Problem für hochqualitative und innovative Forschung, da mit solchen Daten heutzutage kaum mehr publizierbare Artikel zu produzieren sind.

Neben der Transparenzdatenbank ist gerade der Regelungsrahmen der bereits erwähnten Forschungsfinanzierungsdatenbank im Diskussion. Hier sollen alle Forschungsfinanzierungen durch die öffentliche Hand abrufbar sein, auch jene, die an private Unternehmen ausgeschüttet werden, einschließlich der steuerlichen Forschungsprämie. Nach gegenwärtigem Stand sollen der Wissenschaft nur zusammengefasste Daten bereitgestellt werden, aber keine Einzelfalldaten, bei denen die Identifikation einzelner Förderungsempfänger nicht ganz ausgeschlossen werden könnte. Dagegen sollen von der öffentlichen Hand bestellte Evaluatorlnnen von Forschungsförderungsmaßnahmen auf Basis einer konkreten Beauftragung im Einzelfall Zugang zu Einzelfalldaten erhalten, sofern es für die konkrete Fragestellung notwendig und sinnvoll erscheint. Die Entscheidung bleibt also bei der beauftragenden öffentlichen Einrichtung.

Vergleicht man Regelungen zu Registerdaten nach F0G, zu Daten aus dem TDBG und zu Daten aus der FFDB, so sieht man, dass der Gesetzgeber keine einheitliche Vorstellung davon hat, unter welchen Bedingungen die Wissenschaft Zugang zu Einzelfalldaten erhalten soll. Dies zeigt sich auch bei den Bemühungen, der Wissenschaft einen besseren Zugang zu den Daten der Statistik Austria einzuräumen.

4 https://www.parlament.gv.at/PAKT/VHG/XXVI/ME/ME_00010/index.shtml\#tab-Stellungnahmen

5 BGBI. I Nr. 70/2019

6 "Die Möglichkeit des BMF die Daten aus der Transparenzdatenbank in anonymisierter Form an Dritte zu übermitteln, soll dahingehend präzisiert werden, dass dies verpflichtend vorgesehen wird, allerdings mit der Einschränkung für wissenschaftliche Zwecke. Es soll gleicher Zugang für alle wissenschaftlichen Einrichtungen bei voller Transparenz bezüglich Verwendung der Daten und der Ergebnisse gelten. [...." (AA-109 XXVI. Gesetzgebungsperiode, Begründung) 7 Informationszweck, Nachweiszweck, Steuerungszweck, Überprüfungszweck und Wirtschaftlichkeitszweck.

8 Vgl. Erwägungsgrund 26 der Verordnung (EU) 2016/679 des Europäischen Parlaments und des Rates vom 27. April 2016 zum Schutz natürlicher Personen bei der Verarbeitung personenbezogener Daten, zum freien Datenverkehr und zur Aufhebung der Richtlinie 95/46/EG (Datenschutz-Grundverordnung) Etwa bereichsspezifische Personenkennzeichen (bPKs), wie sie das E-Government-Gesetz vorsieht. 


\section{DIE DATEN DER STATISTIK AUSTRIA}

Der größte Datenhalter der öffentlichen Hand in Österreich ist die Statistik Austria. ${ }^{10}$ Dort liegen Kopien eines großen Teils der Daten die auch in den Registern der öffentlichen Hand stehen - und noch viel mehr: Von der Konjunkturerhebung über die Leistungs- und Strukturstatistik der österreichischen Wirtschaft, der F\&E Erhebung, den Erhebungen zum Einsatz von Informations- und Kommunikationstechnologien in Haushalten und Unternehmen, dem Gebäude und Wohnungsregister bis hin zum Krebsregister und der Todesursachenstatistik, um nur einige zu nennen.

Der verbesserte Zugang der Wissenschaft zu den Einzelfalldaten der Statistik Austria würde einen riesigen Datenbestand für die wissenschaftliche Analyse zugänglich machen. Bereits vor zehn Jahren gab es daher - ausgehend von Vertreterlnnen der Wissenschaft (vgl. Gaulhofer, 2009) - Bemühungen, den Zugang der Wissenschaft zu den Datenbeständen der Statistik Austria zu verbessern. Schon damals wollte man eine anstehende Novelle des Bundesstatistikgesetzes nutzen, die Einzelfalldatenbestände der Statistik Austria für die Wissenschaft zu öffnen. Seither haben sich zahlreiche Menschen aus der Wissenschaft, der öffentlichen Verwaltung und auch aus der Statistik Austria für dieses Ziel verwendet (zuletzt etwa Kocher und Badelt, 2019); vor drei Jahren mündeten die Bestrebungen in der Schaffung der "Plattform Registerforschung"."1

2017 und 2018 schien die (nach wie vor erforderliche!) Novellierung des Bundesstatistikgesetzes jeweils knapp bevorzustehen, um dann doch wieder verschoben zu werden. Der damals etablierte Dialog zwischen Politik, Statistik Austria, Stakeholdern und Wissenschaft ist inzwischen wieder zum Erliegen gekommen und das Bundeskanzleramt als zuständiges Ressort arbeitet exklusiv mit Statistik Austria an einer Lösung, ohne die betroffene Wissenschaft oder andere Stakeholder weiter einzubinden. Dem Vernehmen nach ist in Bälde mit einem neuen Vorschlag des Bundeskanzleramts zu rechnen, auch weil eine Novellierung des Bundesstatistikgesetzes im Regierungsübereinkommen vorgesehen ist. Die Knackpunkte sind der Rechtsanspruch auf Zugang der Wissenschaft zu den Einzelfalldaten der Statistik Austria und dessen praktische Durchsetzung, die Verknüpfbarkeit mit anderen Datenbeständen, insbesondere Registerdaten nach FOG sowie die Gewährleistung des Datenschutzes sowie die Kosten des Zugangs zu Einzelfalldaten der Statistik Austria.

\section{AUSTRIAN MICRO DATA CENTER}

Trotz der erzielten Fortschritte, namentlich durch die Ermöglichung der Registerforschung im FOG, hinkt Österreich in puncto Einzelfalldatenzugang der Wissenschaft immer noch weit hinter internationalen Vorbildern wie Dänemark, Schweden oder den Niederlanden her (vgl. Falk-Hölzl-Oberhofer, 2015). Die österreichische Bundesregierung kommt in ihren Forschungs- und Technologieberichten der Jahre 2018 und 2019 zum selben Schluss (siehe Polt, Streicher et al., 2018 und Ecker et al., 2019). Damit ist evidenzbasierte Politik zum Vorteil der Allgemeinheit mangels datengetriebener Analysen weiterhin nicht - oder nur sehr eingeschränkt - möglich.

Ein Plan zur Abhilfe ist unter dem Schlagwort eines sogenannten „Austrian Micro Data Center" immer wieder in Diskussion. Es wurde vom ehemaligen fachstatistischen Generaldirektor der Statistik Austria nach Vorbild des dänischen Mikrodatenzentrums eingebracht. Mit dieser Einrichtung liefert die dänische Statistikbehörde jährlich Daten für rund 400 neue Forschungsprojekte, und setzt dabei umgerechnet etwa vier Millionen Euro um. ${ }^{12}$ Da Dänemark bevölkerungsmäßig etwa halb so groß ist wie Österreich, wird deutlich, welches Potential für den heimischen Wissenschaftsstandort hier schlummert.

Das „Austrian Micro Data Center” würde wie ein One-Stop-Shop funktionieren, der Wissenschafterinnen und Wissenschaftern Zugang zu den Einzelfalldaten der Statistik Austria ebenso ermöglicht wie zu den Registerdaten nach F0G. Zentral ist dabei die Verknüpfbarkeit von Statistik- und Registerdaten miteinander auf Ebene der erfassten Einheiten, also in der Regel auf der Ebene einzelner natürlicher oder juristischer Personen bzw. Unternehmen. Auch die Verschneidung mit externen Daten, etwa Befragungsdaten, ${ }^{13}$ soll möglich sein. Daraus ergäben sich neue Perspektiven, etwa auch für Sozialerhebungen und andere Umfragen. Solche Verknüpfungen verschiedener Datenbestände erfolgen mittels nicht rückführbarer Kennzahlen, um den Datenschutz zu optimieren. Der Zugang zu den Daten würde über einen gesicherten Fernzugriff möglich sein, sodass die Daten nicht heruntergeladen werden können und das „Austrian Micro Data Center“ niemals verlassen.

\section{DATENSCHUTZ, GEHEIMHALTUNG, SENSIBILITÄT UND GESELLSCHAFTLICHE VERANTWORTUNG}

Besonderes Augenmerk liegt beim Zugang der Wissenschaft zu Register- und Statistikdaten auf dem Datenschutz, insbesondere der Geheimhaltung von personen- oder unternehmensbezogenen Daten. In Bezug auf gesundheitsbezogene Daten reagieren Datenschützer und Oppositionsparteien besonders sensibel, wie in Österreich in Zusammenhang mit der Novellierung des FOG festzustellen war (vgl. etwa ede/apa, 2018) und jüngst in Deutschland in Bezug auf eine erweiterte Nutzung von Gesundheitsdaten für Forschungszwecke (dpa, 2019). Die betroffenen Personen scheinen dies weniger dramatisch zu sehen: In Folge der breiten öffentlichen Diskussion um den Zugang der Wissenschaft zu den Daten der Elektronischen Gesundheitsakte (ELGA) meldeten sich geschätzt etwa 5.000 Personen zusätzlich ab (WZ online, APA, 2018). Dies entspricht nicht einmal 0,06 Prozent der im Jahresdurchschnitt 2018 an-

10 Eigentlich Bundesanstalt "Statistik Österreich“ (vgl. § 22 Abs. 1 Bundesstatistikgesetz)

11 Siehe https://www.registerforschung.at

12 Davon ist circa ein Drittel staatliche Basisförderung, der Rest wird den Betreibern der durchgeführten Projekte in Rechnung gestellt.

13 Vgl. etwa die Möglichkeit in den Niederlanden Daten des "Survey of Health, Ageing and Retirement in Europe” (SHARE) mit administrativen Daten zu verknüpfen: http://www.share-project.org/special-data-sets/record-linkage-project/linkage-share-nl.html 
spruchsberechtigten Personen in der österreichischen Krankenversicherung, und ist auch nur ein Bruchteil jener, die sich in den Jahren davor bereits - aus anderen Gründen - von ELGA abgemeldet haben. ${ }^{14}$

Registerdaten und Einzelfalldaten der amtlichen Statistik sind in der Regel nicht vollständig anonymisierbar und das kann datenschutzrechtlich tatsächlich ein ernstzunehmendes Bedenken sein. Allerdings: Die im FOG vorgeschriebene Pseudonymisierung, also das Ersetzen von Namen durch nicht rückführbare Kennzahlen, die (soweit man weiß) auch beim zukünftigen Zugang der Wissenschaft zu Einzelfalldaten der Statistik Austria eingesetzt werden soll und die das Verknüpfen unterschiedlicher Datenbestände erlaubt, ist darauf ausgelegt, Personen und Unternehmen zu schützen, deren Daten in einem Register oder in der amtlichen Statistik erfasst sind. Dies gilt bis auf wenige Ausnahmen.

Um welche Ausnahmen kann es sich hier handeln? Dazu zwei (theoretische) Beispiele: So könnten Wissenschafterinnen und Wissenschafter einzelne Personen, etwa nahe Verwandte oder gute Freunde identifizieren, von denen sie einige kritische Merkmalsausprägungen kennen. Auch Unternehmensdaten könnten dann betroffen sein, wenn es in einzelnen Branchen nur wenige große Unternehmen gibt, die ohne Anführen der Firma anhand der Kombination aus Branche und Beschäftigtenzahl identifizierbar wären

Für diese (seltenen, aber nicht auszuschließenden) Ausnahmen liegt es am Gesetzgeber eine Entscheidung zu treffen. Wir sind der Meinung, dass der potentielle Nutzen der Gesellschaft an den Ergebnissen der Registerforschung höher zu bewerten ist als der mögliche Nachteil, den einzelne Personen erleiden könnten, würden sie über Registerdatensätze identifiziert. Dies aus drei Gründen. Erstens, weil das FOG ohnehin weitreichende Datensicherheitsmaßnahmen vor, um jeglichen Missbrauch zu verhindern (§ $2 \mathrm{~d}$ Abs. $1 \mathrm{FOG}$ ). Außerdem fallen Wissenschafterinnen und Wissenschafter, die den Datenzugang missbrauchen, unter den Strafrahmen der Datenschutz-Grundverordnung. Und auch alle bisher bekanntgewordenen Entwürfe zum Zugang der Wissenschaft zu Einzelfalldaten der Statistik Austria sehen rigide Sicherheitsmaßnahmen vor sowie massive Konsequenzen für Fehlverhalten bis hin zur gerichtlichen Strafbarkeit

Zu den Sicherheitsmaßnahmen gehört es - zweitens - auch, dass im Fall des Austrian Micro Data Centers die Daten nur im Fernzugriff zugänglich sein sollen und dessen Server niemals verlassen würden. Dies würde auch für die Registerdaten nach FOG gelten, wenn sie über das Austrian Micro Data Center verfügbar gemacht werden. Diese strengen Vorgaben zu Sicherheitsmaßnahmen, die Pseudonymisierung und die hohen Strafdrohungen scheinen unseres Erachtens geeignet, das Risiko für Fehlverhalten und Datenmissbrauch effektiv auszuschalten. Dies ist zudem aus den Erfahrungen jener Länder abzuleiten, die der Wissenschaft bereits einen besseren Zugang zu ihren Register- und Einzelfalldaten ermöglicht haben.

Drittens ist hier darauf hinzuweisen, dass sich innerhalb der Wissenschaft die forschungsintrinsischen Maßstäbe verändert haben. Die Forschenden sind bezüglich ethischer Fragestellungen ebenso sensibler geworden, wie grundsätzliche Aspekte der Wissenschaftsintegrität thematisiert werden - und das impliziert die gesellschaftliche Verantwortung. So haben sich auch innerhalb der Wissenschaft Institutionen, Policies und Verfahren zur Klärung ethischer Problemstellungen etabliert, die für die Forschende einen handlungsnormativen Rahmen festlegen. Dazu zählen etwa die Österreichische Agentur für Wissenschaftliche Integrität (OeAWi) oder die an zahlreichen Forschungseinrichtungen mittlerweile etablierten Ethikkommissionen.

\section{KONKRETISIERUNG DES REGIERUNGSPROGRAMMS}

Die neue Bundesregierung sollte aus Sicht der Plattform Registerforschung in der Konkretisierung der im Regierungsprogramm formulierten Vorhaben jedenfalls zu folgenden drei Punkten aktiv werden:

Erstens, der mangelhafte Zugang der Wissenschaft zu Einzelfalldaten der Statistik Austria ist ein hausgemachtes Problem. Dänemark, Schweden oder die Niederlande, in denen der Zugang zu Daten der amtlichen Statistik liberaler geregelt ist, unterliegen demselben europäischen Rechtsrahmen und können daher als Vorbild dienen. Der österreichische Gesetzgeber sollte den Forschenden in diesem Land einen Datenzugang auf internationalem Niveau ermöglichen und damit den Wissenschaftsstandort Österreich stärken.

Erfreulicherweise sieht das aktuelle Regierungsprogramm in diesem Bereich die Einrichtung eines Austrian Micro Data Center vor. Zweitens sollte dieses Center so gestaltet werden, dass es den einfachen und sicheren Zugang der Wissenschaft zu den Einzelfalldaten der Statistik Austria und der Registerdaten nach FOG in einem One-Stop-Shop ermöglicht. Im Zuge der Einrichtung des Centers sollten auch die Bundesministerinnen und Bundesminister ermutigt und aufgefordert werden, jene Registerdaten für die Wissenschaft zu öffnen, die in ihren Ressorts schlummern.

Die Bemühungen sollten sich nicht nur in der Schaffung des erforderlichen legistischen Rahmens erschöpfen, sondern den tatsächlichen Fortschritt laufend überprüfen. Oft sind gesetzliche Regelungen im ersten Wurf noch unausgegoren und müssen nachjustiert werden. Aber auch eine perfekte rechtliche Grundlage ist erst die halbe Miete; sie muss erst von den betroffenen Institutionen gelebt und umgesetzt werden. Dies betrifft die Statistik Austria ebenso wie die Bundesministerien und anderen Einrichtungen, die bundesgesetzlich vorgesehene Register führen. Das Regierungsprogramm bleibt hier auf der Ebene allgemeiner Bekenntnisse. Drittens ist daher als konkreter Schritt auch ein solches Monitoring der Umsetzung der Datenzugangsstrategie zu etablieren.

Mit diesen drei Maßnahmen wäre ein wesentlicher Schritt getan, den Wissenschaftsstandort Österreich abzusichern und zugleich auch wieder näher an jene Länder heranzuführen, die hier inzwischen eine internationale Vorbildfunktion einnehmen. Davon würden natürlich Österreichs wissenschaftliche Einrichtungen und die dort arbeitenden Wissenschafterinnen und Wissenschafter profitieren. Gerade vor dem Hintergrund der Digitalisierung auch weiter Teile der Forschung in den Sozial- und Lebenswissenschaften ist ein gut ausgebauter Datenzugang essentiell für den Standort - auch um hervorragende Forscherlnnen an heimische Institutionen zu rufen bzw. hier im Land zu halten. Zudem tut sich auch auf europäischer Ebene einiges, wie zum Beispiel die kürzlich veröffentliche "Europäische Datenstrategie" (Europäische Kommission

$14 \quad$ Insgesamt waren zum Zeitpunkt des Erscheinens des Artikels im Mai 2018 rund 273.000 Personen von ELGA abgemeldet. Das entspricht etwa 3\% der im Jahresdurchschnitt 2018 anspruchsberechtigten Personen in der österreichischen Krankenversicherung. Siehe Hauptverband der österreichischen Sozialversicherungsträger, Tabelle 3. 
2020), die als wesentlicher Baustein gesehen wird, die europäische Digitalindustrie wieder global wettbewerbsfähig zu machen. Die Vorteile, die sich für Evaluierungen - allgemein aber auch im FTI-Politik-Bereich durch einen gut ausgebauten Datenzugang ergeben, müssen hier nicht weiter erläutert werden.

Aber eben nicht nur die österreichische Wissenschaft würde profitieren: Ein moderner und adäquater Zugang der Wissenschaft zu Registerund Statistikdaten ist die Voraussetzung für erfolgreiche evidenzbasierte Politik zum Nutzen der Allgemeinheit, der Steuerzahlerinnen und Steuerzahler ebenso wie des Wirtschaftsstandorts. Daher fordern wir: Zugang zu den Register- und Individualdaten für die Wissenschaft!

\section{LITERATUR}

Apuzzo, Matt, We Just Wanted to Talk E.U. Farm Policy. Why Was Someone Always Looking Over Our Shoulders?, The New York Times, 3. November 2019, https://www.nytimes.com/2019/11/03/reader-center/ eu-farm-subsidy-reporting.html

Connelly, Roxanne, Dibbend, Chris, Gaylec, Vernon, Playford, Christopher J., The role of administrative data in the big data revolution in social science research, Social Science Research, Volume 59, September 2016, Pages 1-12, https://doi.org/10.1016/j.ssresearch.2016.04.015

dpa, Grüne warnen vor Plänen zu Gesundheitsdaten-Forschung, Handelsblatt, 3. November 2019, https://www.handelsblatt.com/dpa/ wirtschaft-handel-und-finanzen-gruene-warnen-vor-plaenen-zu-gesundheitsdaten-forschung/25184230.html

Ecker, Brigitte et al., Österreichischer Forschungs und Technologiebericht 2019, Lagebericht gem. § 8 (1) FOG über die aus Bundesmitteln geförderte Forschung, Technologie und Innovation in Österreich, Bundesministerium für Bildung, Wissenschaft und Forschung, Bundesministerium für Verkehr, Innovation und Technologie, Bundesministerium für Digitalisierung und Wirtschaftsstandort, 2019, https://www.bmbwf. gv.at/dam/jcr:6c2909b9-d953-4575-8aed-50e705999f8a/Forschungs-_ und_Technologiebericht_2019_dt_bf.pdf

ede/apa, Wer hat Zugriff auf Gesundheitsdaten?, Wiener Zeitung, 11. April 2018, https://www.wienerzeitung.at/nachrichten/politik/oesterreich/958173-Wer-hat-Zugriff-auf-Gesundheitsdaten.html

Europäische Kommission 2020, Mitteilung der Kommission. Eine europäische Datenstrategie, Brüssel 19.2.2020, https://ec.europa.eu/info/ sites/info/files/communication-european-strategy-data-19feb2020_ de.pdf Falk, Martin, Hölzl, Werner, Oberhofer, Harald, Die Bedeutung von unternehmensbezogenen Individualdaten für die empirische Wirtschaftsforschung und wirtschaftspolitische Beratung, Österreichisches Institut für Wirtschaftsforschung, WIFO Monatsberichte, 2015, Jahrgang 88 (11), S. 845-857, https://www.wifo.ac.at/jart/prj3/wifo/resources/ person_dokument/person_dokument.jart?publikationsid $=585228 \mathrm{mi}$ me_type=application/pdf

Gaulhofer, Karl, Wifo-Alarm: Statistik Austria blockiert Forschung, Die Presse, 09.07.2009, https://www.diepresse.com/493556/wifo-alarm-statistik-austria-blockiert-forschung
Hauptverband der österreichischen Sozialversicherungsträger, Versicherte Pensionen Renten, Jahresergebnisse 2018, http://www. sozialversicherung.at/cdscontent/load?contentid=10008.5552018version $=1548666221$

Kocher, Martin G., Badelt, Christoph, Manifest für eine evidenzbasierte Politik, Der Standard, 20. November 2019, https://www.derstandard. at/story/2000111261182/manifest-fuer-eine-evidenzbasierte-politik

Oberhofer, Harald, Schwarz, Gerhard, Strassnig, Michael, Registerforschung: Verwaltungs- und Statistikdaten für die Wissenschaft, Mitteilungen der Vereinigung Österreichischer Bibliothekarinnen und Bibliothekare, Bd. 72, Nr. 2 (2019), D0I: https://doi.org/10.31263/voebm.v72i2.3154, https://journals.univie.ac.at/index.php/voebm/article/ view/3154/2782

Polt, Wolfgang, Streicher, Jürgen et al., Österreichischer Forschungsund Technologiebericht 2018, Bericht der Bundesregierung an den Nationalrat gem. § 8 (2) FOG über die Lage und Bedürfnisse von Forschung, Technologie und Innovation in Österreich, Bundesministerium für Bildung, Wissenschaft und Forschung, Bundesministerium für Verkehr, Innovation und Technologie, Bundesministerium für Digitalisierung und Wirtschaftsstandort, 2018, https://www.bmvit.gv.at/dam/jcr:847e6be5fb89-4b97-b90b-619d84a69538/ftb_2018.pdf

Stanzl, Eva, Forschung, Daten, der Schlüssel zum Wissen, Wiener Zeitung, 13. April 2018, https://www.wienerzeitung.at/nachrichten/wissen/ mensch/958675-Daten-der-Schluessel-zu-Wissen.html?em_no_split=1

WZ online, APA, Tausende Abmeldungen von ELGA, Wiener Zeitung, 6. Mai 2018, https://www.wienerzeitung.at/nachrichten/politik/oesterreich/963188-Tausende-Abmeldungen-von-ELGA.html

\section{AUTOREN}

\section{GERHARD SCHWARZ}

WIFO - Österreichisches Institut für Wirtschaftsforschung

E: gerhard.schwarz@wifo.ac.at

\section{THOMAS KÖNIG}

IHS - Institut für höhere Studien und Wissenschaftliche Forschung

E: koenig@ihs.ac.at

\section{HARALD OBERHOFER}

WU - Wirtschaftsuniversität Wien

E: harald.oberhofer@wu.ac.at

\section{MICHAEL STRASSNIG}

WWTF - Wiener Wissenschafts-, Forschungs- und Technologiefonds E: michael.strassnig@wwtf.at

\section{KEYWORDS:}

Registerforschung, Administrative Daten, Austrian Micro Data Center, Bundesstatistikgesetz, Statistik Austria 